\title{
Supply-Adequacy-Based Optimal Construction of Microgrids in Smart Distribution Systems
}

\author{
S. A. Arefifar, Member, IEEE, Yasser A-R I. Mohamed, Senior Member, IEEE, and T. H. M. EL- \\ Fouly, Member, IEEE
}

\begin{abstract}
Recently, the concept of microgrids (clusters of distributed generation, energy storage units and reactive power sources serving a cluster of distributed loads in grid-connected and isolated grid modes) has gained a lot of interest under the smart grid vision. However, there is a strong need to develop systematic procedure for optimal construction of microgrids. This paper presents systematic and optimized approaches for clustering of the distribution system into a set of virtual microgrids with optimized self-adequacy. The probabilistic characteristics of distributed generation (DG) units are also considered by defining two new probabilistic indices representing real and reactive power of the lines. Next, the advantages of installing both distributed energy storage resources (DESRs) and distributed reactive sources (DRSs) are investigated to improve the self-adequacy of the constructed microgrids. The new strategy facilitates robust infrastructure for smart distribution systems operational control functions, such as self-healing, by using virtual microgrids as building blocks in future distribution systems. The problem formulation and solution algorithms are presented in this paper. The well-known PG\&E 69-bus distribution system is selected as a test case and through several sensitivity studies, the effect of the total DESRs or DRSs capacities on the design and the robustness of the algorithm are investigated.
\end{abstract}

Index Terms-Microgrid design, energy storage devices, reactive sources, energy losses, power imbalance, graph partitioning, tabu search.

\section{INTRODUCTION}

$\mathrm{P}$ OWER system structure has been changed significantly in the last decades. Introduction of renewable energy resources and electric energy storage devices on one hand and the increase in the rate of electricity consumption, associated costs of large power generating plants and environmental issues on the other hand, have manifested the need for new planning, operation and control strategies in the power distribution systems. In this scenario, the interest for local connection of renewable energy resources at the distribution level has gained lots of interest in industry and has changed the conventional distribution systems into multiple modern, interconnected distribution systems, so called microgrids. Microgrids are small electrical distribution systems which connect several electricity consumers to some distributed generators and storage units, which are mainly interfaced by power electronic converters [1].

S. A. Arefifar and T. H. M. El-Fouly are with CanmetENERGY, Natural Resources Canada (NRCan), Varennes QC J3X 1S6, Canada (e-mail: arefifar@ieee.org, telfouly@nrcan.gc.ca).

Yasser A.-R. I. Mohamed is with Department of Electrical and Computer Engineering, University of Alberta, Edmonton, T6G 2V4. (e-mail: yasser_rady@ieee.org)
There are several papers in literature related to microgrids and their potential benefits for the utility and customers [2][5]; however, the concept of how to construct a microgrid and what factors are to be considered in their design has not been addressed properly. In particular, designing microgrids for optimum microgrid supply adequacy requires special attention to facilitate powerful infrastructure for self-healing control under the smart grid environment. Microgrids can be operated in autonomous, grid-connected and ride-through between the two modes [6]-[9]. The less the transferred energy between the microgrids, or the less the generation-load imbalance within them, the more self-sufficient the microgrids will be. Thus, more loads can be supplied in case of autonomous-mode operation of microgrids in the distribution system and the energy losses on power lines connecting the microgrids will be minimized. Therefore, minimization of power imbalance in microgrids or transferred energy between them can be considered as one of the important factors in construction of microgrids.

Distributed generators (DGs) play an important role in construction of microgrids. A strategy to maximize the potential benefits of renewable resources and distributed energy storage devices is to optimally allocate and determine the size of distributed energy storage resources (DESRs) in a distribution system. By increasing the penetration level of renewable resources such as wind turbines or photovoltaic (PV) modules in the system, the intermittent characteristics of them can adversely affect the entire grid. In this situation, the integration of DESRs in the system is one of the feasible solutions to facilitate high level of renewable generation in the system. Moreover, DESRs will accommodate the amount of spilled energy by the renewable resources and this will benefit both utilities and DG owners [10]. The problem of optimally allocating and scheduling DESRs has already been solved in the literature [10]-[16]; however the concept of microgrid is not considered in their formulation and design.

Another approach to enhance the self-adequacy of microgrids is introducing distributed reactive sources (DRSs) to the distribution system. The reactive power can be produced by distributed generators themselves or by capacitor banks. The reactive sources can reduce system losses, improve voltage profiles and in some cases, increase system capacity. In order to get the maximum benefits from DRSs, determination of their best locations and sizes in the system is necessary. Optimal allocating and sizing of distributed reactive sources has been addressed in literature [17]-[22]; however, the microgrid concept again is not considered by the 
authors.

In this paper, a systematic approach is presented for optimal construction of microgrids. For this purpose, initially, a sample system is built up by optimal allocation of different kinds of distributed generation (DG) units in a distribution system. The DGs types are assumed to be of standard types such as wind turbines, PV modules and biomass generators. PV and wind DGs are intermittent, cyclic and weather-based and biomass DG is constant power. Other types of DGs such as the small hydro, CHPs, etc., from the output power perspective, could also be characterized as generators with constant power, cyclical constant power, intermittent, etc., in the same approach that has been done for PV, wind or biomass DGs.

After optimal allocation of DGs, as a utility action, four different scenarios are followed to construct the microgrids. Firstly the system is divided into microgrids by minimizing the total annual transferred energy between microgrids without adding any DESRs or DRSs to the system. Afterwards, the DESRs, DRSs or both, are optimally allocated and sized in the system in order to minimize the objective function. In all cases the objective function is the total annual energy transferred between microgrids or total annual load-generation energy imbalance in the zones. The probabilistic characteristics of DG units are also considered by defining two new probabilistic indices representing real and reactive power of the virtual cut-set lines. As a result, in case of faults or operation in autonomous mode, more loads can be supplied through the distributed generators in each microgrid. Further, the proposed microgrid construction approach facilitates splitting large distribution systems into optimized set of microgrids with high self-adequacy. The new design also facilitates robust infrastructure for smart distribution systems operation and control, such as self-healing control, by using virtual microgrids as building blocks in future distribution systems. The proposed design is in line with the recently developed IEEE Std 1547.4-2011, which presented a microgrid structure as the building blocks of active distribution systems [23].

The optimal allocation of different types of DG units in a large distribution system, presented in this paper, is done with different approaches for a smaller system in [24]-[25]. The main contributions of the paper to the research field are related to the optimum construction of microgrids as follows:

- The development of a systematic strategy for constructing self-sufficient microgrids,

- Taking into account the uncertain characteristics of DG units and loads, for construction of microgrids as a step towards an improved self-healing action and coordinated control actions in a smart grid.

- Optimally allocating and sizing DESRs in the distribution system in order to construct optimum microgrids,

- Optimally allocating and sizing DRSs in the distribution system in order to construct optimum microgrids,

- Simultaneous allocation of DESRs and DRSs in the distribution system for construction of optimum microgrids.
Probabilistic load flow approaches, graph related theories and Tabu search optimization technique are used to conduct this research. Solving this problem will benefit the utility, DG owners and electricity consumers by constructing selfsufficient microgrids in the distribution system through optimally allocating DESRs and DRSs and minimizing the power imbalance in the microgrids.

The paper is organized as follows. Section II presents the concepts of design; and Section III explains the models used for the system components including loads, generators, DESRs and DRSs. The formulation of problem is explained in Section IV. Section V explains the solution algorithms, and Section VI presents the results of optimal allocation of different kind of DGs in the well-known PG\&E 69-bus distribution system. Section VII explains the optimum microgrid construction results and the sensitivity studies. In Section VIII, the robustness of the algorithm to variation of load and DG penetration level is investigated; and finally the paper is concluded in Section IX.

\section{DESIGN CONCEPT}

The goal of this research is to design microgrids in a distribution system including several dispatchable and intermittent distributed generation units for optimum microgrid supply adequacy. Clustering large scale distribution systems into a set of smaller microgrids can have several benefits for the utility, electric power consumers and DG owners. These benefits include easier control strategy, distributed control among microgrids, load routing and transfer among microgrids, enhanced reliability, etc. In response to disturbances, a self-healing system reconfiguration that splits a power network into self-sufficient islands can stop the propagation of disturbances and avoid cascading events. One of the important measures to determine the boundaries of the islands, by the existing system reconfiguration methods, is to minimize the real and reactive power imbalance within the islands [26]. Although the controllable generation and load must be balanced once a system partitions, if we consider minimizing the power imbalance in the zones at the planning stage, balancing generation and load will be much more convenient and effective in the operating stage. If the cut sets are designed properly, each island can behave as a microgrid and may operate with minimum interactions from other parts of the system. This will prevent the disturbances from propagating over all the system, and reduce the affected loads by the disturbance. Therefore, an optimal partitioned system is the one with minimum generation-load imbalance in each zone. Although in some zones the generation level may not meet the required consumption at the peak level, the islanding may be possible at light-load periods. In the worst scenario that the distributed generation penetration level is low, islanding will be possible through minimum load shedding actions and this will be beneficial for both utility and customers. Further, the created virtual microgrids can be controlled in a coordinated manner to optimize the overall system-level operational aspects. 


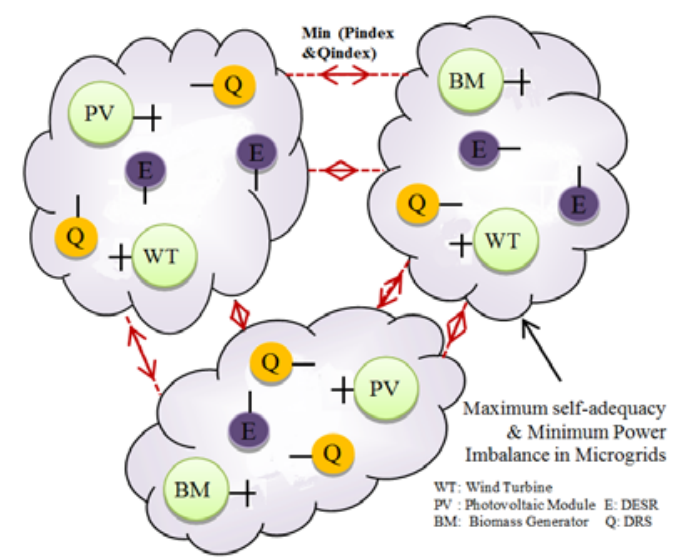

Fig. 1 The main design concepts and the virtual microgrids.

The self-adequacy of microgrids is maximized by minimizing the energy flow between microgrids. If the microgrids are $100 \%$ self-adequate, the energy flow between microgrids is zero and if the microgrids are supplied by the power flowing into them ( $0 \%$ self-adequate), the energy flow between the microgrids is at a maxima. Therefore, the flowing power between the microgrids can be used as an index to represent the self-adequacy of them. The advantage of installing DESRs and DRSs to the distribution system is also considered in order to increase the self-sufficiency of the constructed microgrids. The DESRs can store the otherwise spilled energy generated by renewable-based resources such as wind turbines or even during off-peak hours of system operation while the energy prices are usually lower. Storing the energy and releasing it during on-peak period will increase the self-adequacy of the microgrids. On the other hand, DRSs which could be an independent reactive source or DG connected unit, inject reactive power to the distribution system and decrease the load-generation imbalance in microgrids. Considering the benefits of both DESRs and DRSs, if we install them in the distribution system simultaneously, the constructed microgrids will have less load-generation imbalance, which will be beneficial for both utility and customers. The question here is how to allocate these resources in the distribution system and how to split the distribution system so that the maximum self-adequate microgrids are achieved in the presence of the uncertain nature of renewable resources and loads. This paper answers this concern by optimally allocating the DESRs and DRSs into the distribution system and simultaneously searching for the optimum microgrids in terms of self-adequacy. The special characteristics of distribution system associated with power balancing, such as the intermittent nature of DG units and loads, are considered in the design by applying a probabilistic power balancing approach. The main design concepts proposed in this paper and how the virtual microgrids are clustered are shown in Fig. 1.

\section{Modeling OF LOADS, DGs, DESRs AND DRSs}

In order to optimally design microgrids with maximum self-adequacy, characteristics of all the system components, such as the uncertain nature of DG units and loads, should be modeled properly and be considered in the design. For this research, DG units are modeled as a combination of PV modules, wind turbines and biomass generators, which is the typical combination of most commonly used DG units in distribution systems. Other types of DGs could also be modeled with similar approaches. In this section, the steps taken to model the DG units, loads, DESRs, and DRSs are presented.

\section{A. PV Module Generators}

The output of each PV Module depends on the amount of solar irradiance, ambient temperature and characteristics of the module itself. For this research, the solar irradiance for each hour of the day is modeled by the Beta probability density function (PDF) using historical data [25]. In order to get the PDF of solar irradiance, the data related to a period of one year can be assessed and four days can be selected as representatives of four seasons. The day representing each season is further divided into 24-hour time segments, each having a PDF for solar irradiance. When the solar irradiance is modeled for each hour, the probabilistic function of the output power of PV modules can be generated easily. The Beta PDF used to describe the probabilistic nature of solar irradiance, is as shown in (1).

$$
f_{b}(s)=\frac{\Gamma(\alpha+\beta)}{\Gamma(\alpha) \Gamma(\beta)} \times s^{(\alpha-1)} \times(1-s)^{(\beta-1)} \quad 0 \leq s \leq 1, \alpha \geq 0, \beta \geq 0
$$

where $s$ is solar irradiance $\left(\mathrm{kW} / \mathrm{m}^{2}\right), f_{b}(s)$ is Beta distribution function of $s$, and $\alpha$ and $\beta$ are parameters of the Beta distribution function. The parameters of the Beta distribution function are calculated by using the mean $(\mu)$ and standard deviation $(\sigma)$ of random variable $s$ as shown in (2).

$$
\beta=(1-\mu) \times\left(\frac{\mu \times(1+\mu)}{\sigma^{2}}-1\right) \& \alpha=\frac{\mu \times \beta}{1-\mu}
$$

The Beta PDF is generated for each time segment (one hour for this research) and the probabilistic output power of the PV modules is then calculated using the following equations [25]:

$$
\begin{gathered}
T_{c y}=T_{A}+S_{a y}\left(\frac{N_{O T}-20}{0.8}\right) \\
I_{y}=S_{a y}\left[I_{s c}+K_{i}\left(T_{c}-25\right)\right] \\
V_{y}=V_{o c}-K_{v} \times T_{c y} \\
P S_{y}\left(S_{a y}\right)=N \times F F \times V_{y} \times I_{y} \\
F F=\frac{V_{M P P} \times I_{M P P}}{V_{o c} \times I_{s c}}
\end{gathered}
$$

where $T_{c y}$ and $T_{A}$ are cell and ambient temperature during state $y\left({ }^{\circ} \mathrm{C}\right) ; K_{v}$ and $K_{i}$ are voltage and current temperature coefficients $\left(\mathrm{V} /{ }^{\circ} \mathrm{C}\right.$ and $\left.\mathrm{A} /{ }^{\circ} \mathrm{C}\right) ; N_{O T}$ is nominal operating temperature of cell per ${ }^{\circ} \mathrm{C}$; $F F$ is fill factor; $I_{s c}$ and $V_{o c}$ are short circuit current $(A)$ and open circuit voltage $(V)$; $N$, is the number of cells; $I_{M P P}$ and $V_{M P P}$ are current $(A)$ and voltage $(V)$ at maximum power point.; $P s_{y}$ is the output power of the PV module during state $y$ and $s_{a y}$ is the average solar irradiance of state $y$.

\section{B. Wind Turbine Generators}

The output power of wind turbines depends on the wind speed and parameters of the wind-power conversion curve. For this research, similar to the PV module generators, the wind speed for each hour of the day is modeled by the Weibull PDF by using historical data [26]. In order to get the PDF of 
wind speed, again, four days can be selected as representatives of four seasons. The day representing each season is further divided into 24-hour time segments, each having a probability density function for wind speed. Once the wind speed is modeled for each hour, the probabilistic function of the output power of wind turbines can be generated easily. The wind speed is usually modeled using the Rayleigh PDF which is a special case of the Weibull PDF as shown in (8) [24].

$$
f(v)=\left(\frac{2 v}{c^{2}}\right) \times \exp \left[-\left(\frac{v}{c}\right)^{2}\right]
$$

where $c$ is called the scale index. The mean value of wind speed is calculated using the historical data for each time segment and then the scale index is calculated as shown in (9).

$$
\begin{gathered}
v_{m}=\int_{0}^{\infty} v f(v) d v=\int_{0}^{\infty} v\left(\frac{2 v}{c^{2}}\right) \times \exp \left[-\left(\frac{v}{c}\right)^{2}\right] d v=\frac{\sqrt{\pi}}{2} c \\
c \approx 1.128 v_{m}
\end{gathered}
$$

The output power of a wind turbine is then calculated using (10) [10],

$$
P v_{w}\left(v_{a w}\right)= \begin{cases}0 & 0 \leq v_{a w} \leq v_{c i} \\ P_{\text {rated }} \times \frac{\left(v_{a w}-v_{c i}\right)}{v_{r}-v_{c i}} & v_{c i} \leq v_{a w} \leq v_{r} \\ P_{\text {rated }} & v_{r} \leq v_{a w} \leq v_{c o} \\ 0 & v_{c o} \leq v_{a w}\end{cases}
$$

where $v_{c i}, v_{r}$, and $v_{c o}$ are cut in, rated and cut out speed of the wind turbine, respectively; $P v_{w}$ is the output power of the wind turbine during state $w$; and $v_{a w}$ is the average wind speed of state $w$.

\section{Biomass Generators and Loads}

The biomass generators are assumed to be firm generators with constant output powers without uncertainty. The load model is assumed to follow the IEEE-RTS [27]. In this system the hourly peak load is presented as a percentage of the daily peak load.

\section{DESRs and DRSs Modeling}

The DESRs are modeled as loads during the charging period and as generators during discharging period. It is also assumed that the DESRs charge only during off-peak hours of the day and discharge only during the on-peak hours. Therefore, for each period of 24 hours there is one chargedischarge cycle. A constraint has been introduced on the amount of power generated by the DESRs as shown in (11).

$$
\sum_{i} P_{D E S R_{-} i, t} \leq \sum_{j} P_{\text {Load }_{-} j, t} \quad \forall t
$$

where $P_{\text {Load }_{-} j, t}$ is the real power consumed at bus $j$ at time $t$. This constraint keeps the output power of DESRs always less than the total demand during each hour and guarantees that the reverse power flow is coming from the DG units during any hour. The DRSs are also modeled as simple reactive sources with specific rated capacity.

\section{E. Combined Generation-Load Model}

For this research, a one year period is divided into four seasons and one day is representing each season. Furthermore, each day is divided into 24-hour time segments where each have their own PDFs for the output power of PV modules and wind turbines. In order to integrate the output power of PV modules and wind turbines as multistate variables in the formulations, the continuous PDF of each is divided into different states. The selected number of states affects the accuracy and complexity of the formulation. In this research, the output power of the wind turbine and PV modules for each hour of the day is divided into twelve segments. This means for each hour of the day we have twelve states of solar DG power and twelve states of wind DG power with different probabilities. Assuming that solar irradiance and wind speed states are independent, the probability of any combination of the load and generation is obtained by convolving the two probabilities. Therefore, for each hour we have $144(12 \times 12)$ states with different probabilities and for each day there are $3456(24 \times 144)$ states. In order to get the power and energy losses for the system, the deterministic power flow is run for each state and accumulated considering the probability of each state.

\section{PRoBlem Formulation}

Optimization problems are constructed from an objective function, control variables and some constraints. This section explains the proposed problem formulation and associated constraints for optimal construction of microgrids.

\section{A. Optimization Objective Function}

This research includes two different optimization problems with two different objective functions. It is assumed that there are some candidate buses for installing DGs. These candidate buses are selected according to some parameters such as investor decisions, availability of energy etc. When the candidate buses are determined it is the utilities decision to whether allow the customers to install their DGs in the system or not. The utility will perform this task in a way to get the maximum benefits.

In general maximizing the benefits in any planning problem is minimizing the cost while maintaining the system performance within acceptable limits. Here, the DGs are supposed to be customer owned; therefore, any costs related to the DGs including the capital cost, running cost, etc. is the responsibility of the customers and are not considered in the design. Therefore, the main concern of the utility will be the network losses. The losses are a key consideration in the planning problem because minimizing power losses will relieve the feeders, reduce voltage drop, improve voltage profile and also has other environmental and economical benefits. Also, minimizing losses in DG allocation will avoid the reverse power flow from large DG units that can increase the losses and overheat the feeders [25]. Therefore, the goal of the first problem is to minimize annual energy losses by optimally allocating different kinds of distributed generators. This scenario is applicable in distribution systems without DG units and faced with large number of DG connection applications. It is necessary to find the optimum locations and sizes of DG units under typical DG/load uncertainties. The objective function for the first problem can be defined as (12).

$$
F_{1}=\text { Annual Energy Losses }=\sum_{n=1}^{N} P_{\text {Loss }_{n}} \times \rho_{n} \times h_{n}
$$


where $N$ is the number of states for a year, shown in (13),

$$
\begin{aligned}
N & =365 \times(\# \text { of states per day) } \\
& =365 \times 3456=1,261,440
\end{aligned}
$$

$P_{\text {Loss }_{n}}$ is the system losses for that specific hour of the year, $\rho_{n}$ is the probability of the related state and $h_{n}$ is the time segment of the related state which is one hour for this research. Minimizing the total energy losses of the system is achieved by optimally allocating the DGs. Once this stage is done, the system is ready to be partitioned into microgrids.

The second optimization problem is optimal construction of microgrids. In this case, the total capacities of DESRs and DRSs are assumed to be pre-specified based on economic studies; therefore, the only goal is to minimize the real and reactive power imbalance between generation and loads within the microgrids by properly allocating and sizing of them. This scenario applies to distribution systems with an existing fuel mix of renewable and dispatchable DG units.

To improve the system performance in terms of security of supply and more improved self-healing control actions, under the smart grid vision, the system can be transferred into a set of optimally constructed microgrids by integrating DESRs and/or DRSs. This is a step towards having self-sufficient islands in response to disturbances, which splits the power system into islanded microgrids as a self-healing strategy. Remarkable gain in system supply reliability can be achieved by implementing self-healing control structure in distribution systems. Although self-healing control actions is more general and may include system reconfigurations, load shedding, etc., if the microgrids are designed as self-sufficient zones, selfhealing control actions such as system reconfigurations and load shedding will be done in a more appropriate and effective way to maximize the reliability and robustness of the power infrastructure.

In order to minimize the power imbalance in the microgrids and consider the probabilistic nature of the renewable resources, two new probabilistic indices are defined and presented in the objective function. The objective function for this problem can be formulated as (14):

$$
F_{2}=\frac{a \times P_{\text {index }}+b \times Q_{\text {index }}}{(N o M-1)}
$$

where $P_{\text {index }}$ and $Q_{\text {index }}$ are the defined indices to represent the probabilistic real and reactive power of the virtual cut set lines connecting the microgrids together, and calculated in (15).

$$
P_{\text {index }}=\sum_{k=1}^{N}\left|P_{i j_{k}}\right| \times \rho_{k} \quad \& \quad Q_{\text {index }}=\sum_{k=1}^{N}\left|Q_{i j_{k}}\right| \times \rho_{k}
$$

where $i$ and $j$ are the nodes connecting two microgrids together, $N$ is the number of states for each year (365 $\times 3456$ (states/day)), and $\rho_{k}$ is the probability of the state in which the real and reactive powers are calculated. Selection of factors "a" and "b" depends on the system requirement for balancing the load-generation in the microgrids. Traditionally only the active power has been considered in system partitioning [28]-[31], which means $a=1$ and $b=0$. As an example, in [28] it is claimed that local reactive power compensators can be used to compensate reactive power imbalance. However, [26], by considering the reactive power as an important role player in supporting the voltage profile and knowing that a significant mismatch of reactive power supply and demand causes high or low voltage conditions within islands, has considered both real and reactive power balance in system partitioning. In conclusion "a" and "b" should be selected so that the power imbalance in the subsystems remains minimal and the system constraints such as voltage limits in the sub-systems are met. Since only the ratio of them is important for minimization, "a" and "b" could be set as equal ( 0.5 each in this research). NoM is the number of constructed microgrids and the denominator is set to normalize the cost function. The power balance objective function can be calculated by performing a probabilistic power flow in the system.

\section{B. Optimization Constraints}

There are several assumptions and constraints for solving the two problems defined in this paper. For the optimal allocation of DGs, the following constraints exist, which relates to the distribution system and DG limitations.

- Penetration level of different types of DG, as w\% of feeder capacity for wind based power, s\% of feeder capacity for solar power and b\% of feeder capacity for biomass generation,

$$
\begin{aligned}
& \sum P_{D G_{-} W T}=\% \text { w of feeder capacity } \\
& \sum P_{D G_{-} P V}=\% \text { of feeder capacity } \\
& \sum P_{D G_{-} B M}=\% \text { of feeder capacity }
\end{aligned}
$$

Some distribution system companies may have limitations on the percentage of distributed generators allowed in their systems. For instance in Canada, according to [24], local distribution companies in Ontario were required to accept a percentage of customer owned wind-based DG units in their systems.

- Power flow equations,

$$
\begin{aligned}
& P_{\text {Sub }_{t}}+\sum P_{D G_{t}}-\sum P_{\text {Load }_{t}}= \\
& \sum_{i=1}^{\text {nbus }} V_{t, i} \times V_{t, j} \times Y_{i, j} \times \cos \left(\theta_{i j}+\delta_{t, j}-\delta_{t, i}\right) \quad \forall t \\
& Q_{S u b_{t}}+\sum Q_{D G_{t}}-\sum Q_{\text {Load }_{t}}= \\
& -\sum_{i=1}^{n b u s} V_{t, i} \times V_{t, j} \times Y_{i, j} \times \sin \left(\theta_{i j}+\delta_{t, j}-\delta_{t, i}\right) \quad \forall t
\end{aligned}
$$

-Voltage limits at all the system buses including the slack bus,

$$
\begin{gathered}
V_{\text {min }} \leq V_{t, i} \leq V_{\max } \quad \forall i \neq 1, \\
V_{t, 1}=1 \angle 0
\end{gathered}
$$

- In a distribution system with large improperly allocated DG units, the reverse power flows from DG units to the power system through the substation transformer. This reverse current increases the losses and can overheat feeders; therefore, the utility has specific overriding rights to curtail the output of the DGs to limit this reverse power flow to $60 \%$ of substation rating [10]. 


$$
-0.6 \times P_{\text {rated }} \leq P_{\text {sub }} \leq P_{\text {rated }}
$$

For the second optimization problem, other than the above mentioned items, the total energy capacity of DESRs and their power ratings, as well as total reactive power of DRSs should be considered as follows:

$$
\begin{gathered}
\sum P_{D E S R s_{-} i}=P_{D E S R} \quad \sum E_{D E S R s_{-} i}=E_{D E S R} \\
\sum Q_{D R S_{\_} i}=Q_{D R S}
\end{gathered}
$$

Further to the above limitations, the Power flow equations should be modified in order to consider the real power charged or discharged by the DESRs and also the reactive power generated by the DRSs as shown in (23) and (24).

$$
\begin{gathered}
P_{\text {Sub }}+\sum P_{D G_{t}} \pm \sum P_{D E S R s}-\sum P_{\text {Load }_{t}}= \\
\sum_{i=1}^{n b u s} V_{t, i} \times V_{t, j} \times Y_{i, j} \times \cos \left(\theta_{i j}+\delta_{t, j}-\delta_{t, i}\right) \quad \forall t \\
Q_{S u b_{t}}+\sum Q_{D G_{t}}+\sum Q_{D R S S}-\sum Q_{\text {Load }_{t}}= \\
-\sum_{i=1}^{\text {nbus }} V_{t, i} \times V_{t, j} \times Y_{i, j} \times \sin \left(\theta_{i j}+\delta_{t, j}-\delta_{t, i}\right) \quad \forall t
\end{gathered}
$$

The power of DESR units is positive in the discharging period and negative in the charging period.

\section{Optimization Control Variables}

For the optimal allocation of different kinds of DGs, the control variables are locations and sizes of different DGs in the system. For the optimal construction of microgrids, the control variables are divided into two sets, 1) the locations and sizes of the DESRs and DRSs in the distribution system and 2) the virtual cut set lines that connect the microgrids together. These parameters will be adjusted using optimization techniques explained in the next section to get the optimum results.

\section{SOLUTION AlgORITHMS}

The problem formulated in Section IV is a comprehensive multi-objective problem. Further, the combinatorial nature of the present problem demands efficient solution algorithms. Heuristic optimization techniques are well-suited for such optimization problems. In this paper, three different types of algorithms are used at different stages, including Tabu Search (TS) optimization algorithm as the main optimization method and graph theory-related algorithms as well as forwardbackward-based probabilistic power flow method. The flowchart of the algorithms is shown in Fig. 2.

The TS Algorithm is a heuristic search method that uses different memory structures to guide the search to a good solution both economically and effectively [32]-[33]. In this section the solution algorithm to main problem which is to construct the optimum microgrids by inserting DESRs and DRSs to the distribution system is explained. In the following subsections, the steps taken for implementing the algorithms are presented.

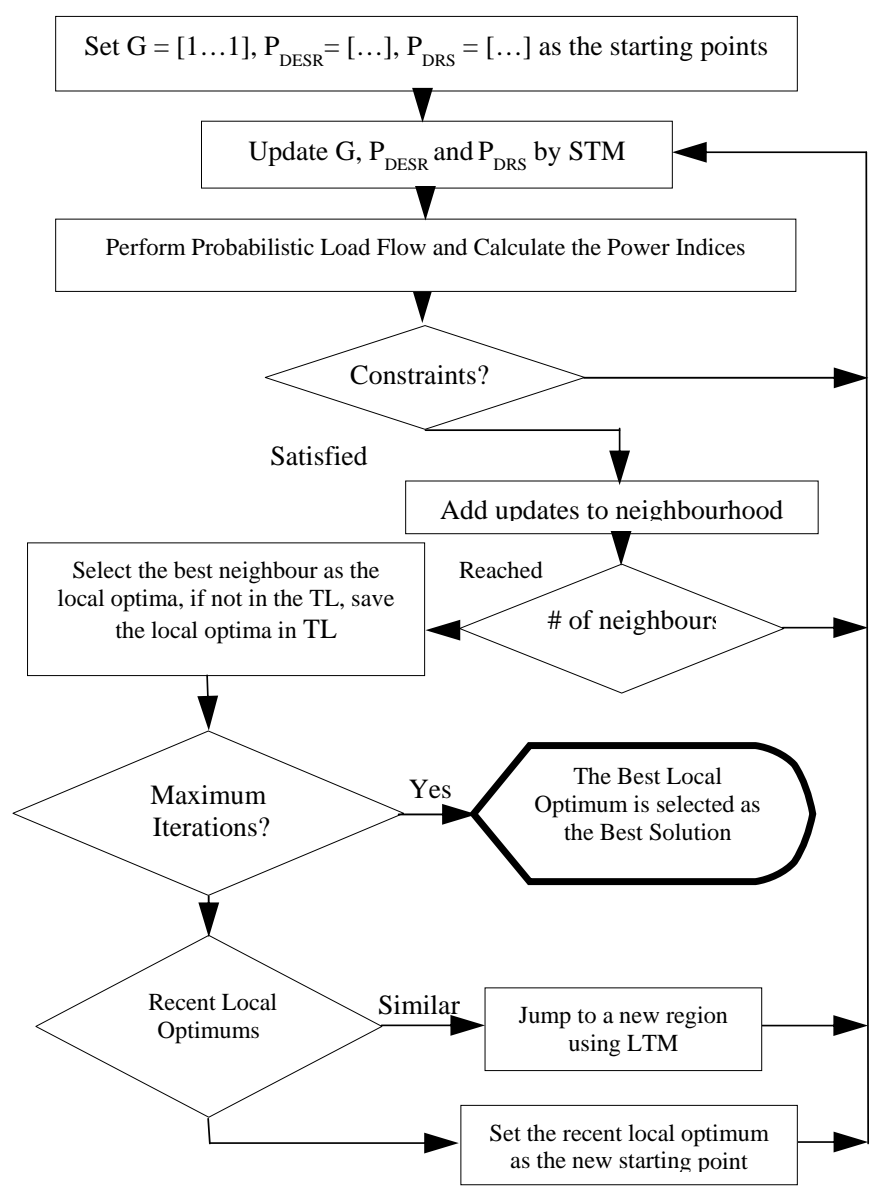

Fig. 2 Flowchart of the algorithms used for the design.

\section{A. Neighborhood Definition in Tabu Search (TS)}

The TS starts with a feasible solution and continues iteratively until certain criterion, which is usually the maximum iteration numbers, is reached. The control variables to make the neighbors are the locations and sizes of the DESRs and DRSs in the distribution system as well as the virtual cut set lines that connect the microgrids together. These control variables for each system can be represented as the following vectors:

$$
\begin{aligned}
& P_{D E S R}=\left[\begin{array}{llll}
P_{D E S R_{-} I} & \ldots \ldots & P_{D E S R_{-} k}
\end{array}\right], \\
& P_{D R S}=\left[\begin{array}{llll}
P_{D R S} 1 & \ldots & \ldots & P_{D R S} k_{k}
\end{array}\right],
\end{aligned}
$$

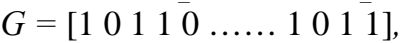

where the length of the vectors $P_{\mathrm{DESR}}, P_{\mathrm{DRS}}$ and $G$ is equal to the number of buses and the power lines which can be considered as cutting edges respectively. Each derivative of $P_{D E S R}$ and $P_{D R S}$ represents the rated capacity of DESRs or DRSs in that specific bus. The derivatives of the $G$ vector represent one physical line in the system. The number " 1 " and " 0 " for each derivative represent an inside or between microgrids line respectively. The next step is to make three sets of neighbors for the starting points. A neighbor can be defined in many ways; here we have selected each neighbor by changing some derivatives (e.g. 5 derivatives) of each vector and checking the feasibility of the resulted design in terms of the constraints. There are some constraints for each feasible solution including those mentioned in Section IV-B, 
which were related to power aspects, as well as the graphrelated constraints explained in Section IV-D. The next step is to calculate the objective function for all neighborhoods, set the best neighbor as the new starting point and continue the process.

\section{B. Tabu List and Aspiration}

To avoid stopping in a local optima, and to prevent cycling around it, some Tabu restrictions should be imposed by using a list called Tabu List (TL). This list which has a first in first out structure keeps the best solutions that have been visited in previous moves or the moves that have been resulted in the optimum point in previous regions and avoids revisiting them. The length of TL depends on the size of the problem and is usually determined experimentally. In our problem, we have made the TL from the best recently visited solutions. For this purpose a quantity, which is unique for each set of parameters, and calculated using (28), is saved in the TL.

$$
\sum_{i=1}^{n} G(i) \times 2^{i}+\sum_{i=n+1}^{n+k} P_{D E R}(i) \times 2^{i}+\sum_{i=n+k+1}^{n+2 k} P_{D R S}(i) \times 2^{i}
$$

An aspiration criterion is a rule which releases the members of Tabu list. This relaxation is allowed when the newly met solution point has better properties than the optimum point reached so far. This phenomenon will make the search process more intelligent and avoids missing higher quality results.

\section{Intensification and Diversification in TS}

Two memory structures are used in TS to avoid random search, namely they are Short-Term Memory (STM) and Long-Term Memory (LTM). The STM memorizes the common features of sub-optimal solutions for a number of iterations and then tries to search for the optimum point with similar features in that region. This intensification process will guide the search in each region to the high quality solutions rather than random undirected movements.

During the search process, the LTM is used to diversify the search by jumping to a new region and allow the algorithm to go through all the possible solutions to find the global optima. This long-term memory will keep track of the common features of all initial starting points in different regions to avoid restarting from similar previously used starting points.

The two types of memories have been implemented by using two sets of vectors with the same length as $P_{D E S R}, P_{D R S}$ and $G$. Each derivative of the STM vectors will represent the number of times the associated derivative has been changed in the suboptimal solutions. The larger each derivative indicates the less chance to be the optimum value. Thus, the next movements or neighborhoods will be selected so that the derivatives that are less suspected to be the optimum have higher chance to be changed. The long-term memory is also implemented in a similar way.

\section{Graph-Theory-Related Algorithms}

To check the constraints related to the system topology, some graph-related algorithms are implemented. These algorithms will check whether all the system buses are considered in different microgrids and secondly, if all the buses located in each microgrid are connected together as a tree. In order to answer these two questions, the shortest path algorithm can be used. The shortest path algorithm finds a path between two vertices (buses) in the system such that the sum of weights of its constituent edges is minimized [34]. The impedance of each branch is used as weight of edges in this research. There are several differences depending on whether the given graph is undirected, directed, or mixed. Here, since the main focus is to check the connectivity of the nodes in sub-graphs, we can assume undirected graph, which is generated from distribution system topology.

Several algorithms are proposed to solve shortest path problem. In this paper the Dijkstra's algorithm, which solves the single-source shortest path problems, has been used [35]. To answer the raised questions, the distance from every system bus to all system buses is checked for each suggested microgrid design. If for a specific bus there is at least one path to another bus of the system, then we can conclude that specific bus is considered in a microgrid. If the same criterion applies for all system buses and there were no unconnected buses, this means all the system buses are considered in different microgrids and also they are connected in each microgrid as a tree and the two conditions are satisfied.

\section{E. Forward-Backward-Based Probabilistic Power Flow}

The Forward-Backward load flow algorithm is a well-known power flow method used for radial distribution systems. It is based on the direct application of the KVL and KCL [36] to overcome numerical issues associated with other load flow solution algorithms (e.g. Newton-Raphson) when applied to sparse or weakly-meshed distribution systems. The algorithm starts with assuming a flat profile for voltages of all the buses and follows an iterative-based procedure of three steps: 1) calculation of bus currents, 2) backward sweep, to calculate the branch currents and 3) forward sweep, to calculate the bus voltages. These three steps are repeated until convergence is achieved [36]. To calculate the annual energy losses or the power balance related indices, the forward backward power flow is run for each load-generation state and the results are accumulated considering the probability of states.

In the next sections, the proposed algorithms have been applied to a 69-bus distribution system and several sensitivity studies are performed for the formulation and robustness of the design, and the results are presented.

\section{Optimal Allocation of DGs IN Distribution System}

The well-known PG\&E 69-bus distribution system [37] is selected as a test system for optimal allocation/sizing of distributed generators, designing the optimum microgrids and sensitivity studies. For this research, a typical combination of three types of DG units is assumed; namely, wind-based, solar-based and biomass generators. It is assumed that total rated powers of the wind turbines is $350 \mathrm{~kW}(\mathrm{w}=9.21 \%)$, the total rated powers of the PV modules is $175 \mathrm{~kW}$ ( $\mathrm{s}=4.61 \%$ ), whereas the total rated powers of the biomass generators is $550 \mathrm{~kW}$ ( $\mathrm{b}=14.47 \%$ ). The maximum rated power of each DG unit is set as $100 \mathrm{~kW}$ for wind turbines, $50 \mathrm{~kW}$ for PV modules 
TABLE I. CANDIDATE BUSES FOR INSTALLING DGS

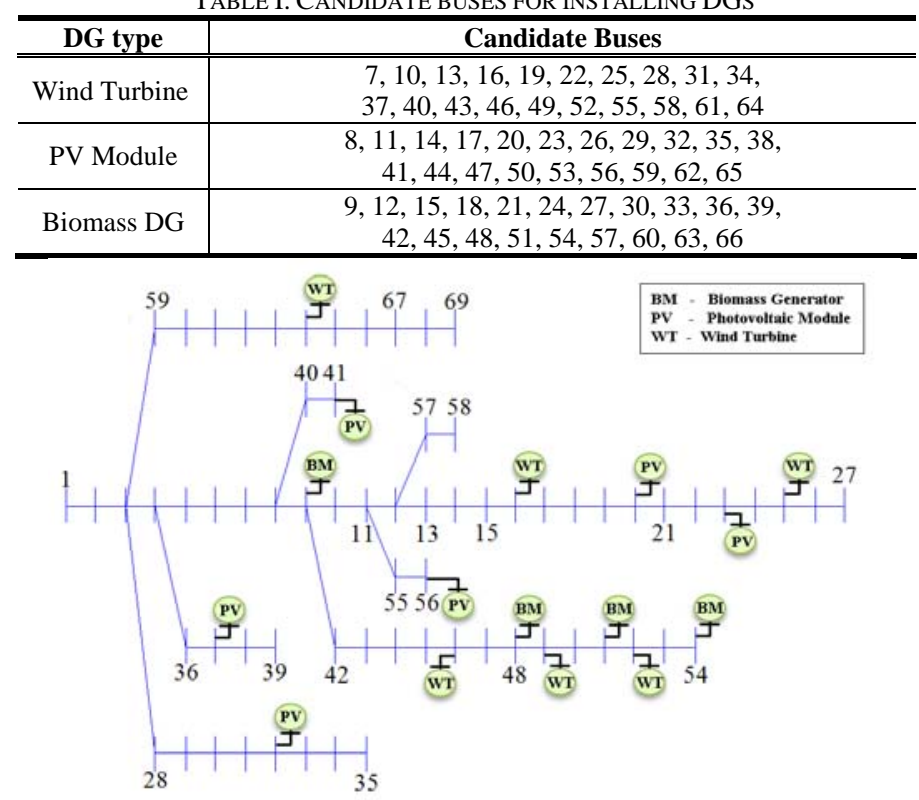

Fig. 3 The 69-bus Distribution system with DGs' locations.

and $200 \mathrm{~kW}$ for biomass generators. The candidate buses for installing wind turbines, PV modules and biomass generators are shown in Table I. It is noted that the candidate buses criterion is not restrictive as it can be increased to include the overall system buses. It is added to adapt the optimization scenario to practical cases, where wind, PV, biomass generators cannot be installed anywhere in the system due to the urbanity, environmental or other aspects of the system under study.

The optimal DG allocation algorithm explained in Section IV is applied in order to get the optimum locations and optimum rated capacities of different types of DGs. As shown in Fig. 3, different kinds of DG units are distributed over the system buses and more concentrated close to the larger loads (whose locations are shown in Fig.4). The rated powers of the DG units are presented in Table II. After installing DG units with the total rated capacity of $28.27 \%$ of total real power load, the annual energy losses of the system is reduced from 1416.1MWh to 867.66MWh. The optimal DG allocation step will be neglected if the distribution system has already installed the DG units.

\section{OPTIMAL MiCROGRID DESIGN}

In this section, the algorithms explained in Section $\mathrm{V}$ are applied to construct the optimum microgrids in the distribution system. For this purpose, the system designed in Section VI is chosen as the base system and then the optimum microgrids are designed for the system in four different scenarios. At the first step, the microgrids are constructed without adding any DESRs or DRSs in the distribution system. Then the DESRs and DRSs, individually and mutually, are allocated in the system to construct optimum microgrids. For each case, the total rated capacities of DESRs and DRSs are changed in order to see its effect on the optimum constructed microgrids and the objective function.

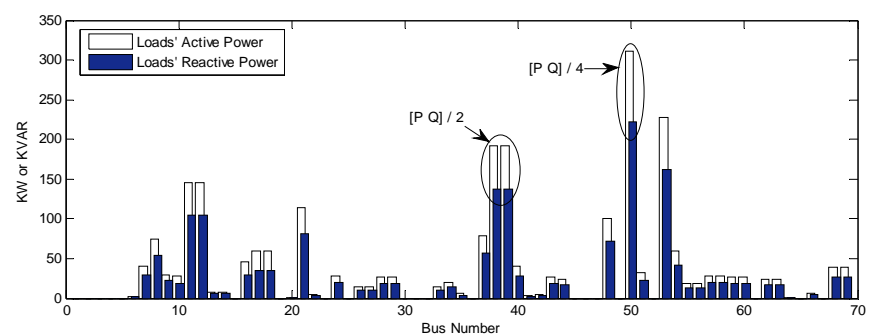

Fig. 4 The 69-bus Distribution system’s loads.

TABLE II. OPTIMUM SELECTED BUSES FOR INSTALLING DGS

\begin{tabular}{c|c|c}
\hline DG type & $\begin{array}{c}\text { Optimum Selected } \\
\text { Buses }\end{array}$ & $\begin{array}{c}\text { Rated DG Capacities } \\
\text { (kW) }\end{array}$ \\
\hline \hline Wind Turbine & $16,25,46,49,52,64$ & $50,25,75,75,100,25$ \\
\hline \hline PV Module & $20,23,37,32,41,56$ & $25,25,25,50,25,25$ \\
\hline \hline Biomass DG & $9,48,51,54$ & $50,125,175,200$ \\
\hline
\end{tabular}

TABLE III. OPTIMAL CONSTRUCTED MICROGRIDS FOR CASE A

\begin{tabular}{c|c|c}
\hline Min NoM & Optimum Virtual Cut Set Lines & $\begin{array}{c}\text { Objective } \\
\text { Function }\end{array}$ \\
\hline \hline 5 & $12,19,28,62$ & 116.1694 \\
\hline 6 & $9,12,19,28,62$ & 199.3942 \\
\hline 7 & $9,12,19,28,47,62$ & 322.8392 \\
\hline 8 & $8,9,12,19,28,47,62$ & 499.7940 \\
\hline 9 & $3,8,9,12,19,28,47,62$ & 718.3084 \\
\hline
\end{tabular}

\section{A. System Partitioning without DESRs and DRSs}

The distribution system with optimum design for the locations and rated capacities of the DG units is partitioned into microgrids at this stage without installing DESRs or DRSs in the system. In this case, mathematically, the optimum result will occur when the system is split into two virtual microgrids and the connecting power line has the minimum transferring energy. In order to have more feasible values (more than two microgrids), another constraint is added, at this stage only, which is on the number of microgrids ( $N o M)$ to be equal or more than X. Table III shows the optimum virtual cut set lines to construct the microgrids while $\mathrm{X}$ varies between 5 and 9. The optimum constructed microgrids for this case where $\mathrm{X}$ is set as 8 is shown in Fig. 5. In Table III, the cut set line $j$ is the line between bus $j$ and $j+1$. It is seen that the system partitioning results and the objective function will be different based on selection of minimum number of constructed microgrids.

\section{B. System Partitioning with DESRs Only}

The distributed energy storage resources are introduced to the system to reduce the negative impact of the intermittent nature of renewable resources. They are also beneficial in terms of saving the amount of spilled energy generated by wind turbines as well as PV modules. In this part, the DESRs are optimally allocated in the system and the system is partitioned simultaneously in order to minimize the loadgeneration imbalance in the constructed microgrids. For this purpose, it is assumed that the total rated power of the DESRs is constant for each case and there is no limit to their stored energy (high energy application). 


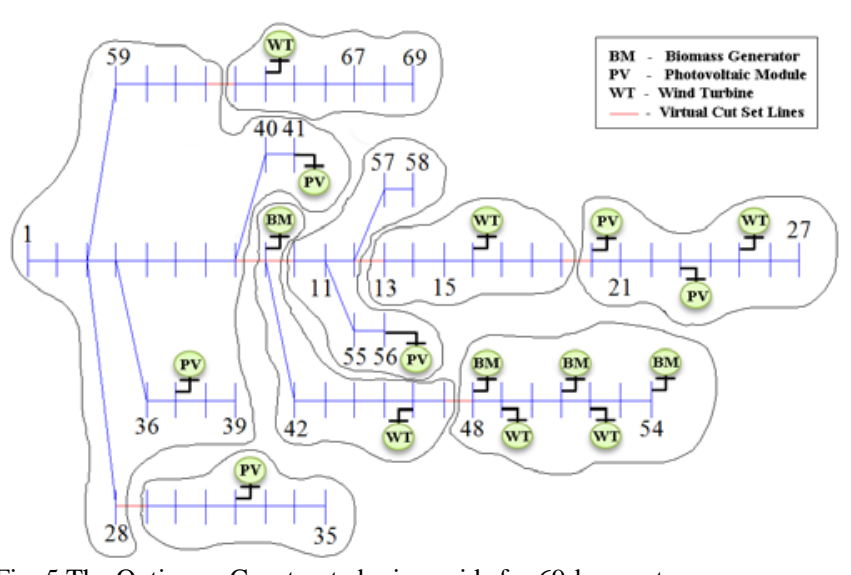

Fig. 5 The Optimum Constructed microgrids for 69-bus system

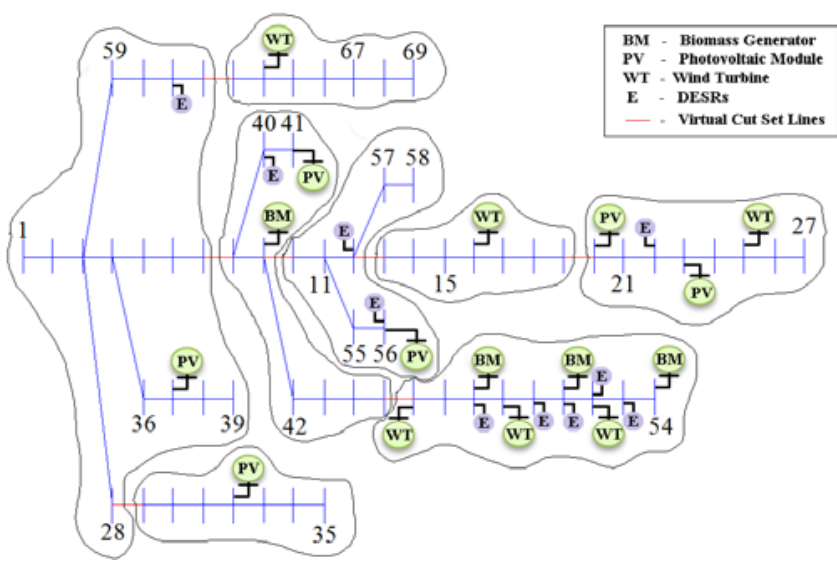

Fig. 6 The Optimum Constructed microgrids with DESRs locations

TABLE IV. OPTIMAL CONSTRUCTED MICROGRIDS FOR CASE B

\begin{tabular}{|c|c|c|c|}
\hline $\begin{array}{l}\mathbf{P}_{\text {DESRS }} \\
(\mathrm{kW})\end{array}$ & Parameter & Results & \begin{tabular}{|l} 
Objective \\
Function
\end{tabular} \\
\hline \multirow{3}{*}{200} & Buses & $18,50,51,52,53$ & \multirow{3}{*}{494.6148} \\
\hline & $\mathbf{P}_{\text {DESR (kW) }}$ & $25,100,25,25,25$ & \\
\hline & Cut Sets & $7,9,12,19,28,45,62$ & \\
\hline \multirow{3}{*}{300} & Buses & $12,19,40,48,50,51,54$ & \multirow{3}{*}{494.6043} \\
\hline & $\mathbf{P}_{\text {DESR }(k W)}$ & $25,25,25,25,25,150,25$ & \\
\hline & Cut Sets & $7,9,12,19,28,45,62$ & \\
\hline \multirow{3}{*}{350} & Buses & $12,22,40,48,50,51,52,53,56,61$ & \multirow{3}{*}{494.6008} \\
\hline & $\mathbf{P}_{\text {DESR }(\mathrm{kW})}$ & $25,25,25,25,100,25,50,25,25,25$ & \\
\hline & Cut Sets & $7,9,12,19,28,45,62$ & \\
\hline \multirow{3}{*}{400} & Buses & $4,9,11,12,19,40,44,50,51,53$ & \multirow{3}{*}{494.6002} \\
\hline & $P_{\text {DESR (kW) }}$ & $25,25,25,25,25,25,50,125,50,25$ & \\
\hline & Cut Sets & $7,9,12,19,28,45,62$ & \\
\hline \multirow{3}{*}{500} & Buses & $5,8,11,17,40,41,47,50,52,53,57,61$ & \multirow{3}{*}{494.5979} \\
\hline & $\mathbf{P}_{\text {DESR }(k W)}$ & $25,50,25,25,25,75,25,150,25,25,25,25$ & \\
\hline & Cut Sets & $7,9,12,19,28,45,62$ & \\
\hline
\end{tabular}

The optimum locations and sizes of DESRs as well as optimum virtual cut set lines and the objective function is presented in Table IV. The optimally constructed microgrids for the case that the total rated capacities of DESRs is set as $350 \mathrm{~kW}$, is shown in Fig. 6.

It is seen that by inserting the DESRs into the system, the objective function which is the energy imbalance in the microgrids is reduced. Since the DESRs do not generate active power, their effects on the total transferred power between the microgrids and the objective function are not as much as DRSs. The optimal constructed microgrids will be affected by introducing DESRs to the system. Also the locations and sizes of DESRs vary for different total rated capacities of DESRs.

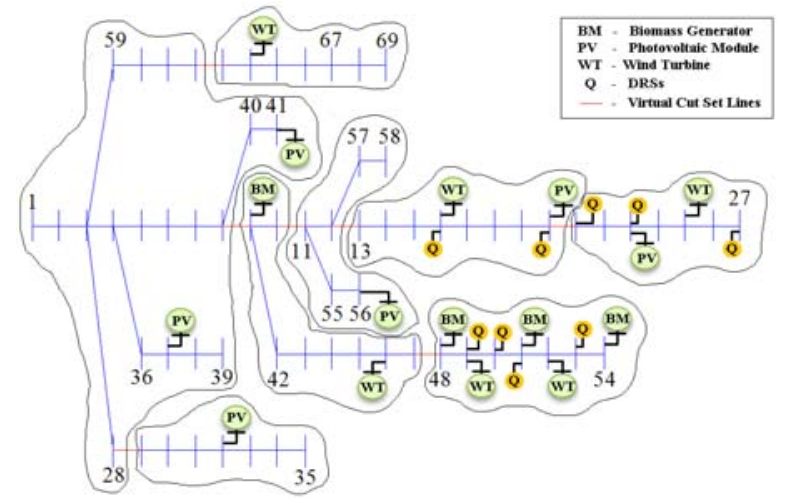

Fig. 7 The Optimal Constructed microgrids with DRSs locations

TABLE V. OPTIMAL CONSTRUCTED MICROGRIDS FOR CASE C

\begin{tabular}{|c|c|c|c|}
\hline $\begin{array}{r}\mathbf{Q}_{\text {DRAs }} \\
(\mathbf{k V N a r})\end{array}$ & Parameter & Results & $\begin{array}{l}\text { Objective } \\
\text { Function }\end{array}$ \\
\hline \multirow{3}{*}{200} & Buses & $14,16,19,23,24,26,27$ & \multirow{3}{*}{447.1635} \\
\hline & $\overline{\text { Q }_{\text {DRS(kVAr) }}}$ & $25,50,25,25,25,25,25$ & \\
\hline & Cut Sets & $7,9,12,19,28,45,62$ & \\
\hline \multirow{3}{*}{300} & Buses & 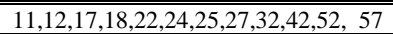 & \multirow{3}{*}{437.7200} \\
\hline & $\mathbf{Q}_{\text {DRS(kVAr) }}$ & $25,25,25,25,25,25,25,25,25,25,25,25$ & \\
\hline & Cut Sets & $8,9,12,19,28,47,62$ & \\
\hline \multirow{3}{*}{350} & Buses & $\begin{array}{l}12,17,18,20,21,24,26,49,50,53 \\
\end{array}$ & \multirow{3}{*}{421.5605} \\
\hline & $\overline{\text { QRRS(kVAr) }}$ & $25,25,25,50,50,25,25,75,25,25$ & \\
\hline & Cut Sets & $8,10,13,20,28,47,62$ & \\
\hline \multirow{3}{*}{400} & Buses & $\begin{array}{l}16,20,21,23,27,49,50,51,53 \\
\end{array}$ & \multirow{3}{*}{413.8431} \\
\hline & $\mathbf{Q}_{\text {DRS(kVAr) }}$ & $25,75,25,25,50,25,25,100,50$ & \\
\hline & Cut Sets & $8,10,13,20,28,47,62$ & \\
\hline \multirow{3}{*}{500} & Buses & 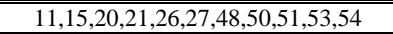 & \multirow{3}{*}{399.416} \\
\hline & QDRS(kVAr) & $50,50,25,25,50,50,50,25,50,25,100$ & \\
\hline & Cut Sets & $8,10,13,20,28,47,62$ & \\
\hline
\end{tabular}

C. System Partitioning with DRSs Only

DRSs are added to the system to reduce losses, improve voltage profile or in some cases increase system capacity [38]. The reactive power can be generated with capacitor banks or as an auxiliary service provided by the distributed generators. In this part, the DRSs are optimally allocated in the system and the system is partitioned in order to minimize the loadgeneration imbalance in the constructed microgrids. Similar to Case B, it is assumed that the total rated capacity of the DRSs is constant for each case. The optimum locations and sizes of DRSs as well as optimum virtual cut set lines and the objective function is presented in Table V. The optimal constructed microgrids for the case that the total rated capacities of DRSs is set as $400 \mathrm{kVAr}$, is shown in Fig. 7. It is seen that by inserting the DRSs into the system, the objective function which is the energy imbalance in the microgrids is reduced. The optimally constructed microgrids and the locations and sized of DRSs also varies for different total rated capacities of DRSs.

\section{System Partitioning with Both DESRs and DRSs}

At this stage, both DESRs and DRSs are added to the system simultaneously and at the same time the system is partitioned into several microgrids in order to minimize the total loadgeneration imbalance in the zones. The system partitioning results related to this section is shown in Table VI. From the results of Cases B and C one can conclude that the total objective function, which is the energy imbalance in the microgrids, will be reduced by adding DESRs or DRSs to the distribution system. 
TABLE VI. OPTIMAL CONSTRUCTED MICROGRIDS FOR CASE D

\begin{tabular}{|c|c|c|c|c|}
\hline $\begin{array}{c}\mathbf{P}_{\text {DESRs }} \\
(\mathrm{kW})\end{array}$ & $\begin{array}{c}\mathbf{P}_{\text {DRSs }} \\
\text { (kVAr) }\end{array}$ & Parameter & Results & $\begin{array}{l}\text { Objective } \\
\text { Function }\end{array}$ \\
\hline \multirow{5}{*}{300} & \multirow{5}{*}{500} & Buses & "19,28,36,37,40,52,56,65,67 & \multirow{5}{*}{399.05} \\
\hline & & $\mathbf{P}_{\text {DESR (kW) }}$ & $25,25,50,25,25,25,50,50,25$ & \\
\hline & & Buses & $15,16,20,24,27,49,50,51,52,53,55$ & \\
\hline & & $\mathbf{Q}_{\text {DRS(kVAr) }}$ & $25,25,50,50,50,50,75,50,75,25,25$ & \\
\hline & & Cut Sets & $8,10,13,20,28,47,62$ & \\
\hline \multirow{5}{*}{350} & \multirow{5}{*}{400} & Buses & 17,23,28,29,36,38,44,52,66,67 & \multirow{5}{*}{414.19} \\
\hline & & $\mathbf{P}_{\text {DESR }(\mathrm{kW})}$ & $25,25,25,25,50,50,75,25,25,25$ & \\
\hline & & Buses & $14,15,20,21,23,25,26,27,48,49,51,53,54,55$ & \\
\hline & & $\mathbf{Q}_{\text {DRS(kVAr) }}$ & $25,25,25,25,25,25,25,25,25,25,50,50,25,25$ & \\
\hline & & Cut Sets & $8,10,13,20,28,47,62$ & \\
\hline \multirow{5}{*}{400} & \multirow{5}{*}{350} & Buses & 6 6,10,17,24,26,28,37,50,52,55,59,61,64 & \multirow{5}{*}{421.17} \\
\hline & & $\mathbf{P}_{\text {DESR (kW) }}$ & $25,25,25,25,25,25,25,50,50,25,50,25,25$ & \\
\hline & & Buses & $19,20,21,23,27,48,50,52,53,54$ & \\
\hline & & $\mathbf{Q}_{\text {DRS(kVAr) }}$ & $50,50,25,50,25,25,25,50,25,25$ & \\
\hline & & Cut Sets & $8,10,13,20,28,47,62$ & \\
\hline \multirow{5}{*}{500} & \multirow{5}{*}{300} & Buses & $\begin{array}{c}6,7,8,12,14,21,26,27,35,38 \\
40,46,47,50,52,58\end{array}$ & \multirow{5}{*}{428.77} \\
\hline & & $\mathbf{P}_{\text {DESR (kW) }}$ & $\begin{array}{c}50,25,25,25,25,25,25,25,25,25 \\
25,50,25,25,50,50 \\
\end{array}$ & \\
\hline & & Buses & $19,20,22,24,50,51$ & \\
\hline & & QDRS(kVAr) $_{\text {D }}$ & $25,75,75,25,50,50$ & \\
\hline & & Cut Sets & $8,10,13,20,28,47,62$ & \\
\hline
\end{tabular}

Table VI also confirms that by adding both DESRs and DRSs into the system and partitioning the system, the magnitude of objective function will reduce more and subsequently, the constructed microgrids are more robust and self-sufficient in case of system faults or unexpected interruptions. As an example the optimally constructed microgrids for the case that the total rated capacities of DESRs and DRSs is set as $350 \mathrm{~kW}$ and $400 \mathrm{kVAr}$, respectively, is shown in Fig. 8. It shows that the DESRs and DRSs are more focused in the microgrids having larger loads.

\section{RoBUStNESS OF THE DESIGN}

The research presented in this paper is related to the planning stage; however, in a real distribution system, the load level usually increases during a long term period of time. On the other hand, the generation level of DG units may vary depending on the types of DG units and uncertainty in their penetration level during a period of time. How these changes will affect the optimal constructed microgrids and whether the planned design is still optimal or near optimal design for the current system are investigated in this section. For this purpose, several case studies are presented by considering different load and generation levels and their effects on the optimum design is observed. The studies are performed for the load and generation changing from $90 \%$ to $150 \%$ and the results are shown in Table VII. For the sensitivity studies in this section it is assumed that the optimum microgrids are designed with adding both DESRs and DRSs to the distribution system. The total rated capacities of DESRs and DRSs are $350 \mathrm{~kW}$ and $400 \mathrm{kVAr}$ respectively. Table VII reveals that although there is a little difference in the optimum infrastructure in each case, the final constructed microgrids is very similar. The objective function, however, which depends on the generation and load levels varies in each case and is the optima for the related system. This means that if for any reason the load or generation changed over a period of time, the designed plan is still valid and close to optima in terms of load-generation imbalance in the microgrids. It is clear that

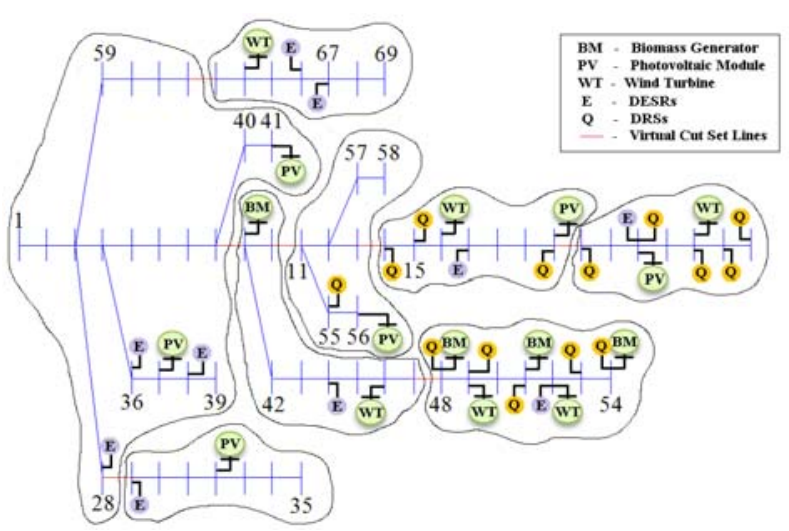

Fig. 8 The Optimal Constructed microgrids with DESRs and DRSs locations

\begin{tabular}{c|c|c|c}
\hline $\begin{array}{c}\text { Load } \\
\mathbf{\%}\end{array}$ & $\begin{array}{c}\text { DG } \\
\mathbf{\%}\end{array}$ & Optimum Virtual Cut Set Lines & $\begin{array}{c}\text { Objective } \\
\text { Function }\end{array}$ \\
\hline \hline 90 & 100 & $7,10,13,20,28,45,62$ & 367.8075 \\
\hline 110 & 100 & $8,10,13,20,28,47,62$ & 472.6284 \\
\hline 120 & 100 & $8,9,12,19,28,47,62$ & 536.3547 \\
\hline 150 & 100 & $8,9,12,19,28,47,62$ & 719.5456 \\
\hline 100 & 90 & $8,10,13,20,28,47,62$ & 424.3959 \\
\hline 100 & 110 & $8,10,13,20,28,47,62$ & 404.0490 \\
\hline 100 & 120 & $7,10,13,20,28,45,62$ & 405.6867 \\
\hline 100 & 150 & $7,10,13,20,28,45,62$ & 374.6481 \\
\hline
\end{tabular}

further increase in the load or penetration level of DGs will affect the optimum design. In such cases, the optimally constructed microgrids should be updated accordingly. It can be noted also that the probabilistic nature of the proposed planning algorithms inherently increases the robustness of the planning problem against load/generation uncertainties.

\section{CONCLUSIONS}

This paper presented a novel strategy for constructing microgrids with optimized supply adequacy. The active distribution system is initially developed by introducing different types of DG units such as wind turbines, PV modules and biomass generators. Considering the probabilistic nature of these resources and the hourly load profile, these generators are optimally sized and allocated in order to minimize the total annual energy losses in the distribution system. Next, by taking advantage of DESRs and DRSs, the system is partitioned into microgrids by optimally sizing and allocating these devices in the system and simultaneously minimizing the energy imbalance in the microgrids. The optimal partitioning scenario in case of emergency is the one with minimum power and energy imbalance in each zone. This will ease the control strategy in each island and protects other healthy areas of the network from the disturbances as a self-healing action in a distribution system.

Several sensitivity studies are conducted on the PG\&E 69bus test system to assess the effects of adding different capacities of DESRs and DRSs into the system. The sensitivity studies show that the optimal constructed microgrids are dependent on the amount of DESRs and DRSs added to the system. The robustness of the optimum infrastructure to the variation of DG penetration level and load 
level is also investigated. The results show that the final result is not very sensitive to the DG and load level, which can be attributed to the probabilistic nature of the proposed planning scheme. The proposed planning framework can help utility engineers and system planners in designing self-sufficient microgrids, which can be a useful step towards the realization of more reliable and sustainable smart distribution grids.

\section{ACKNOWLEDGMENTS}

The authors would like to thank Natural Resources Canada, the Government of Canada for financially supporting this research through the Program on Energy Research and Development.

\section{REFERENCES}

[1] J. Carrasco, L. Franquelo, J. Bialasiewicz, E. Galvan, R. Guisado, M. Prats, J. Leon, and N. Moreno-Alfonso, "Power electronic systems for the grid integration of renewable energy sources: A survey," IEEE Trans. Power Electron., vol. 53, no. 4, pp. 1002-1016, Aug. 2006.

[2] M. Shahabi, M. R. Haghifam, M. Mohamadian, and S. A. Nabavi-Niaki, "Microgrid dynamic performance improvement using a doubly fed induction wind generator,” IEEE Trans. Energy Convers. , vol. 24, no. 1, pp. 137-145, Mar. 2009.

[3] H. Kanchev, L. Di, F. Colas, V. Lazarov, and B. Francois, "Energy management and operational planning of a microgrid with a PV-based active generator for smart grid applications,” IEEE Trans. Ind. Electron., vol. 58, no. 10, pp. 4583-4592, Oct. 2011.

[4] R. Majumder, A. Ghosh, G. Ledwich, and F. Zare, "Load sharing and power quality enhanced operation of a distributed microgrid," IET Renewable Power Generation, vol. 3, no. 2, pp. 109-119, Jun. 2009.

[5] L. H. Lasseter, "Smart distribution: Coupled microgrids," Proc. IEEE, vol. 99, no. 6, pp. 1074-1082, Jun. 2011.

[6] J. Driesen and F. Katiraei, "Design for distributed energy resources," IEEE Power Energy Mag., vol. 6, no. 3, pp. 30-40, May/Jun. 2008.

[7] R. Wai, W. Wang, and C. Lin, "High-performance stand-alone photovoltaic generation system,” IEEE Trans. Ind. Electron., vol. 55, no. 1, pp. 240-250, Jan. 2008.

[8] J. Guerrero, L. Hang, and J. Uceda, "Control of distributed uninterruptible power supply systems," IEEE Trans. Ind. Electron., vol. 55, no. 8, pp. 2845-2859, Aug. 2008.

[9] Y. A.-R. I. Mohamed, H. Zeineldin, M. Salama, and R. Seethapathy, "Seamless formation and robust operation of distributed generation micro-grids via robust direct voltage vector control and optimized dynamic power sharing," IEEE Transactions on Power Electronics, vol. 27, no. 3, pp. 1283-1294, March 2012.

[10] Y. M. Atwa and E. F. El-Saadany, "Optimal allocation of ESS in distribution systems with a high penetration of wind energy," IEEE Trans. Power Syst., vol. 25, no. 4, pp. 1815-1822, Nov. 2010.

[11] P. D. Brown, J. A. Lopes, and M. A. Matos, "Optimization of pumped storage capacity in an isolated power system with large renewable penetration,” IEEE Trans. Power Syst., vol. 23, no. 2, pp. 523-531, May 2008.

[12] C. Lo andM. Anderson, "Economic dispatch and optimal sizing of battery energy storage systems in utility load-leveling operations," IEEE Trans. Energy Convers., vol. 14, no. 3, pp. 824-829, Sep. 1999.

[13] E. Koutroulis, D. Kolokotsa, A. Potrirakis, and K. Kalaitzakis, "Methodology for optimal sizing of stand-alone photovoltaic/wind generator systems using genetic algorithms," Sol. Energy, vol. 80, no. 9, pp. 1072-1088, 2006.

[14] A. Prasad and E. Natarajan, "Optimization of integrated photovoltaic wind power generation systems with battery storage,” Energy, vol. 31, no. 12, pp. 1607-1618, 2006.

[15] F. Galiana, F. Bouffard, J. Arroyo, and J. Restrepo, "Scheduling and pricing of coupled energy and primary, secondary, and tertiary reserves,” Proc. IEEE, vol. 93, no. 11, pp. 1970-1983, Nov. 2005.

[16] C. Abbey and G. Joós, "A stochastic optimization approach to rating of energy storage systems in wind-diesel isolated grids,” IEEE Trans. Power Syst., vol. 24, no. 2, pp. 418-426, Feb. 2009.
[17] A. H. Etemadi and M. Fotuhi-Firuzabad, "Distribution system reliability enhancement using optimal capacitor placement," IET Generation, Transmission \& Distrib., vol. 2, no. 5, pp. 621-631, Sep. 2008.

[18] A. A. Eajal and M. E. El-Hawary, "Optimal capacitor placement and sizing in unbalanced distribution systems with harmonics consideration using particle swarm optimization,” IEEE Trans. Power Del. , vol. 25, no. 3, pp. 1734-1741, Jul. 2010.

[19] H.-D. Chiang, J.-C.Wang, J. Tong, and G. Darling, “Optimal capacitor placement, replacement and control in large-scale unbalanced distribution systems: Modeling and a new formulation,” IEEE Trans. Power Syst., vol. 10, no. 1, pp. 356-362, Feb. 1995.

[20] M. Delfanti, G. P. Granelli, P. Marannino, andM.Montagna, "Optimal capacitor placement using deterministic and genetic algorithms," IEEE Trans. Power Syst., vol. 15, no. 3, pp. 1041-1046, Aug. 2000.

[21] R. A. Gallego, A. J. Monticelli, and R. Romero, "Optimal capacitor placement in radial distribution networks," IEEE Trans. Power Syst., vol. 16, no. 4, pp. 630-637, Nov. 2001.

[22] M. A. S. Masoum, M. Ladjevardi, A. Jafarian, and E. F. Fuchs, “Optimal placement, replacement and sizing of capacitor banks in distorted distribution networks by genetic algorithms," IEEE Trans. Power De, vol. 19, no. 4, pp. 1794-1801, Oct. 2004.

[23] IEEE Guide for Design, Operation, and Integration of Distributed Resource Island Systems With Electric Power Systems, , Jul. 20, 2011, IEEE Std 1547.4-2011.

[24] Y. M. Atwa and E. F. El-Saadany, "Probabilistic approach for optimal allocation of wind-based distributed generation in distribution systems," IET Renewable Power Generation, vol. 5, no. 1, pp. 79-88, Jan. 2011.

[25] Y. M. Atwa, E. F. El-Saadany, M. M. A. Salama, and R. Seethpathy, "Optimal renewable resources mix for distribution system energy loss minimization,” IEEE Trans. Power Syst., vol. 25, no. 1, pp. 360-370, Feb. 2010.

[26] J. Li, C.-C. Liu, and K. P. Schneider, "Controlled partitioning of a power network considering real and reactive power balance," IEEE Trans. Smart Grid, vol. 1, no. 3, pp. 261-269, Dec. 2010.

[27] J. M. S. Pinheiro, C. R. R. Dornellas, and A. C. G. Melo, "Probing the new IEEE reliability test system (RTS-96): HL-II assessment,” IEEE Trans. Power Syst., vol. 13, pp. 171-171, 1998.

[28] K. Sun, D. Zheng, and Q. Lu, "Splitting strategies for islanding operation of large-scale power systems using OBDD-based methods," IEEE Trans. Power Syst., vol. 18, no. 2, pp. 912-923, May 2003.

[29] H. You, V. Vittal, and X. Wang, "Slow coherency-based islanding," IEEE Trans. Power Syst., vol. 19, no. 1, pp. 483-491, Feb. 2004.

[30] B. Yang, V. Vittal, and G. T. Heydt, "Slow coherency based controlled islanding-A demonstration of the approach on the blackout August 14 2003 scenario ," IEEE Trans. Power Syst., vol. 21, no. 4, pp. 18401847, Nov. 2006.

[31] G. Xu and V. Vittal, "Slow coherency based cutset determination algorithm for large power systems,” IEEE Trans. Power Syst., vol. 25, no. 2, pp. 877-884, May 2010.

[32] F. Glover, “Tabu search-Part I," ORSA J. Comput., vol. 1, no. 3, pp. 190-206, 1989.

[33] F. Glover, "Tabu search—Part II," ORSA J. Comput., vol. 2, no. 1, pp. 4-32, 1990.

[34] V. Cherkassky Boris, V. Goldberg Andrew, and R. Tomasz, "Shortest paths algorithms: theory and experimental evaluation," Math. Program.. Ser. A , vol. 73, no. 2, pp. 129-174, 1996.

[35] E. W. Dijkstra, "A note on two problems in connection with graphs," Numerische Mathematik, vol. 1, pp. 269-271, 1959.

[36] D. Shirmohammadi, H.W. Hong, A. Semlyen, and G. X. Luo, "A compensation-based power flow method for weakly meshed distribution and transmission networks," IEEE Trans. Power Syst., vol. 3, no. 2, pp. 753-762, May 1988.

[37] M. E. Baran and F. F. Wu, "Optimal capacitor placement on radial distribution systems," IEEE Trans. Power Del., vol. 14, no. 1, pp. 725734.

[38] M. E. H. Golshan and S. A. Arefifar, "Distributed generation, reactive sources and network-configuration planning for power and energy-loss reduction," Generation, Transmission, Distrib., IET Proc., vol. 153, no. 2, pp. 127-136, Mar. 2006. 
Seyed Ali Arefifar (S'06, M'10) was born in Isfahan, Iran. He received the B.Sc. and M.Sc. degrees with honors in electrical engineering, power systems, from Isfahan University of Technology, Iran, in 2001 and 2004, respectively, and the Ph.D. degree in electrical engineering from University of Alberta, Canada, in 2010. He is currently an NSERC Visiting Fellow at CanmetENERGY, Natural Resources Canada (NRCan), and pursuing the Postdoctoral Program at University of Alberta, Canada. His research interests include optimizations in planning and operation of smart grids, load modeling and power quality.

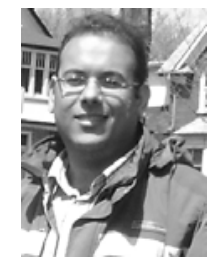

Yasser Abdel-Rady I. Mohamed (M’06, SM’011) was born in Cairo, Egypt, on November 25, 1977. He received the B.Sc. (with honors) and M.Sc. degrees in electrical engineering from Ain Shams University, Cairo, in 2000 and 2004, respectively, and the Ph.D. degree in electrical engineering from the University of Waterloo, Waterloo, ON, Canada, in 2008. He is currently with the Department of Electrical and Computer Engineering, University of Alberta, AB, Canada, as an Assistant Professor. His research interests include dynamics and controls of power converters; distributed and renewable generation; modeling, analysis and control of smart grids; electric machines and motor drives.

Dr. Mohamed is an Associate Editor of the IEEE Transactions on Industrial Electronics. He is also a Guest Editor of the IEEE Transactions on Industrial Electronics Special Section on "Distributed Generation and Micro-grids". His biography is listed in Marque's Who is Who in the World.

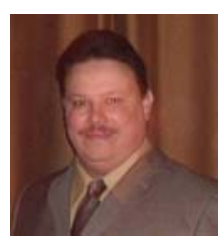

Tarek H.M. EL-Fouly (S'97 - M'07) received his B.Sc. and M.Sc. degrees in Electrical Engineering from Ain Shams University, Cairo, Egypt, in 1996 and 2002, respectively. He received his Ph.D. degree in Electrical Engineering from the University Of Waterloo, Waterloo, Ontario, Canada, in 2008. He joined CanmetENERGY, Natural Resources Canada, in 2008, as a Transmission and Distribution Research Engineer, where he is conducting and managing research activities related to active distribution networks, microgrids and remote communities. In 2010, he was appointed as Adjunct Assistant Professor at the Electrical and Computer Engineering Department at the University of Waterloo. His research interests include protection and coordination studies, integration of renewable energy resources, smart microgrid, smart remote community applications, demand side management and forecasting. 